\title{
Electronic structure of isolated single vacancy centres in silicon carbide
}

\begin{abstract}
The lowest electronic levels of the various charged states of the isolated carbon and silicon single vacancies in silicon carbide have been calculated using the defect molecule approach without lattice distortion.
\end{abstract}

The electronic structure of the isolated carbon and silicon single vacancies in silicon carbide has been investigated using the defect molecule approach developed by Coulson and Kearsley (1957). For this nearest neighbour model an isolated vacancy in the $6 \mathrm{H}$ and $3 \mathrm{C}$ silicon carbide polytypes has the same tetrahedral environment. The single positive, $\mathrm{V}^{+}$, neutral, $\mathrm{V}^{0}$, single negative, $\mathrm{V}^{-}$, and double negatively charged, $\mathrm{V}^{2-}$, states have been considered here.

The method used is similar to that employed by Larkins (1969) for calculations on the isolated vacancy in diamond and in silicon. Atomic functions obtained by Clementi (1965) were used to approximate the $\mathrm{s}$ and $\mathrm{p}$ valence orbitals on the silicon and carbon atoms in the crystal. The model assumes that the vacancy is undistorted with the four atoms neighbouring the defect in the $\mathrm{sp}^{3}$ hybridized state. The vacancy electrons are considered to be localized within the defect. However, the exchange contribution between these vacancy electrons and the other bonded valance electrons associated with the four nearest neighbours is included in the potential term. This term is evaluated explicitly instead of using the approximation of Geoppert-Mayer and Sklar (1938). A semiempirical estimate for the one-centre Coulomb integral $Q,\left(Q=\left\langle a(1) a(2)\left|\left(1 / r_{12}\right)\right| a(1) a(2)\right\rangle\right.$, where $a$ is the initial tetrahedral hybrid orbital for the vacancy electron) was used, as well as the value obtained from the analytic function. The data given by Skinner and Pritchard (1953) was used with the procedure outlined by Coulson and Kearsley (1957), to obtained a semiempirical estimate. For both systems the semiempirical value for $Q$ is considerably smaller than the analytic value. Table 1 summarizes the predicted lowest electronic levels for the various isolated vacancy centres in silicon carbide. All levels within $0.5 \mathrm{eV}$ of the ground state are included. The electron-electron interaction is important, and there is strong configurational mixing.

Table 1. Lowest electronic levels for the isolated vacancy centres in silicon carbide

\begin{tabular}{lcccc} 
& \multicolumn{2}{c}{ Silicon vacancy } & \multicolumn{2}{c}{ Carbon vacancy } \\
Centre & & & & \\
& $Q=17.81 \mathrm{eV}$ & $Q=12.86 \mathrm{eV}$ & $Q=11.45 \mathrm{eV}$ & $Q=8 \cdot 10 \mathrm{eV}$ \\
$\mathrm{V}^{+}$ & ${ }^{2} \mathrm{~T}_{2}$ & ${ }^{2} \mathrm{~T}_{2}$ & ${ }^{2} \mathrm{~T}_{2}$ & ${ }^{2} \mathrm{~T}_{2}$ \\
$\mathrm{~V}^{0}$ & ${ }^{1} \mathrm{E}^{3} \mathrm{P}_{1}$ & ${ }^{1} \mathrm{E}_{1}{ }^{3} \mathrm{~T}_{1}$ & ${ }^{3} \mathrm{~T}_{1},{ }^{5} \mathrm{~A}_{2}$ & ${ }^{1} \mathrm{~T}_{2},{ }^{1} \mathrm{~A}_{1},{ }^{3} \mathrm{E}$ \\
$\mathrm{V}^{-}$ & ${ }^{4} \mathrm{~A}_{2}$ & ${ }^{4} \mathrm{~A}_{2}$ & ${ }^{4} \mathrm{~A}_{2}$ & ${ }^{2} \mathrm{~T}_{2}$ \\
$\mathrm{~V}^{2}-$ & ${ }^{3} \mathrm{~T}_{1},{ }^{1} \mathrm{E}$ & ${ }^{3} \mathrm{~T}_{1},{ }^{1} \mathrm{E}$ & ${ }^{3} \mathrm{~T}_{1}$ & ${ }^{1} \mathrm{~A}_{1}$
\end{tabular}

For the silicon vacancy the relative ordering of the lowest levels of the various centres is not sensitive to the value of $Q$. Thus the ${ }^{1} \mathrm{E}$ electronic level is predicted to be the ground state of the neutral centre, $0.28 \mathrm{eV}(Q=17.81 \mathrm{eV})$ or $0.22 \mathrm{eV}(Q=12.85 \mathrm{eV})$ below the ${ }^{3} \mathrm{~T}_{1}$ level. For the $\mathrm{V}^{2}-$ centre the ${ }^{3} \mathrm{~T}_{1}$ level is lowest, $0.01 \mathrm{eV}(Q=17.81 \mathrm{eV})$ or $0.12 \mathrm{eV}$ $(Q=12.86 \mathrm{eV})$ below the ${ }^{1} \mathrm{E}$ level. However, the ordering of the levels predicted for the carbon vacancy centres is sensitive to the value of $Q$. The predicted ground state for the neutral centre, when $Q$ has the value $11.45 \mathrm{eV}$, is the ${ }^{3} \mathrm{~T}_{1}$ level $0.16 \mathrm{eV}$ below the ${ }^{5} \mathrm{~A}_{2}$ level; however, for $Q=8.10 \mathrm{eV}$ the ${ }^{1} \mathrm{~T}_{2}$ level is lowest, $0.18 \mathrm{eV}$ and $0.20 \mathrm{eV}$ below the ${ }^{1} \mathrm{~A}_{1}$ and 
${ }^{3} \mathrm{E}$ levels respectively. The ${ }^{4} \mathrm{~A}_{2}$ level of the $\mathrm{V}^{-}$centre is lowest in the unmodified case whereas the ${ }^{2} T_{2}$ level is predicted to be the ground state using the semiempirical estimate. The calculations for the $\mathrm{V}^{2}-$ centre predict that the ${ }^{3} \mathrm{~T}_{1}$ level is lowest for $Q=11.45 \mathrm{eV}$ while the ${ }^{1} \mathrm{~A}_{1}$ level is the ground state for the modified case. Previous calculations on the isolated vacancy in silicon (Larkins 1969) suggest that the predictions using the modified value of $Q$ agree more closely with experimental findings. It is probable that this is also true for these centres.

The energy levels have not been corrected for energy changes due to symmetric relaxation and Jahn-Teller distortion effects. Calculations to first order on the diamond and silicon systems (Larkins 1969) suggest that while the separation between the levels may be sensitive to these effects there is little change in the relative ordering of the levels. The modified calculation for the carbon vacancy centres suggests that there are spin- and orbitally- allowed transitions associated with the $\mathrm{V}^{+}, \mathrm{V}^{0}$ and $\mathrm{V}^{-}$centres which may have absorption energies in the visible region. The energy level separations, neglecting possible lattice distortion or delocalization corrections, are as follows: $\mathrm{V}^{+},{ }^{2} \mathrm{~T}_{2} \rightarrow{ }^{2} \mathrm{E}, 1.23 \mathrm{eV}$; $\mathrm{V}^{0},{ }^{1} \mathrm{~T}_{2} \rightarrow{ }^{1} \mathrm{E}, 2.81 \mathrm{eV},{ }^{1} \mathrm{~A}_{1} \rightarrow{ }^{1} \mathrm{~T}_{2}, 2.44 \mathrm{eV}$, or ${ }^{3} \mathrm{E} \rightarrow{ }^{3} \mathrm{~T}_{1}, 2.39 \mathrm{eV} ; \mathrm{V}^{-},{ }^{2} \mathrm{~T}_{2} \rightarrow{ }^{2} \mathrm{~A}_{1}, 2.07 \mathrm{eV}$. These results will be discussed more fully in a later publication. We are not aware of any experimental results to compare with the findings.

Theoretical Physics Division,

Building 8.9,

Atomic Energy Research Establishment,

Harwell,

Didcot, Berkshire,

England, UK.
F. P. LARKINS $\dagger$

A. M. STONEHAM

14th May 1970

Clementi, E., 1965, Suppl. IBM J. Res. Dev., 9, 2-19.

Coulston, C. A., and Kearsley, M. J., 1957, Proc. R. Soc., A241, 433-54.

GoEPPERT-MAYer, M., and Sklar, A. L., 1938, J. chem. Phys., 6, 645-52.

Larkins, F. P., 1969, D. Phil. Thesis, Oxford, to be published.

Skinner, H. A., and Pritchard, H. D., 1953, Trans. Faraday Soc., 49, 1254-62.

\title{
Correlation functions of a point defect
}

\begin{abstract}
The correlation functions associated with the atoms constituting a point defect can be expressed in terms of the Green functions corresponding to the perturbed atoms by using the pseudomolecular model. An expression for the Debye-Waller factor of a nearest neighbour of a vacancy in the simple cubic lattice is given as an example.
\end{abstract}

The displacement and velocity correlation functions of atoms in the imperfect crystal can be written as (Maradudin 1969)

$$
\begin{aligned}
& \left\langle u(l ; t) u\left(l^{\prime} ; 0\right)\right\rangle=\frac{\hbar}{\pi} \int_{-\infty}^{\infty} \mathrm{d} \omega \exp (-\mathrm{i} \omega t) \frac{\operatorname{sgn} \omega}{\exp (-\beta \hbar \omega)-1} \operatorname{Im} G^{\prime}\left(\boldsymbol{l} ; \boldsymbol{l}^{\prime} ; \omega^{2}-\mathrm{i} \epsilon\right) \\
& \left\langle v(l ; t) v\left(\boldsymbol{l}^{\prime} ; 0\right)\right\rangle=\frac{\hbar}{\pi} \int_{-\infty}^{+\infty} \mathrm{d} \omega \exp (-\mathrm{i} \omega t) \frac{\omega^{2} \operatorname{sgn} \omega}{\exp (-\beta \hbar \omega)-1} \operatorname{Im} G^{\prime}\left(\boldsymbol{l} ; \boldsymbol{l}^{\prime} ; \omega^{2}-\mathrm{i} \epsilon\right)
\end{aligned}
$$

$\dagger$ Also at Mathematical Institute, Oxford. 